\title{
A Specific Role for the Thalamus in Mediating the Interaction of Attention and Arousal in Humans
}

\author{
C. M. Portas, G. Rees, A. M. Howseman, O. Josephs, R. Turner, and C. D. Frith \\ Wellcome Department of Cognitive Neurology, Institute of Neurology, WC1N 3BG, London, United Kingdom
}

The physiological basis for the interaction of selective attention and arousal is not clearly understood. Here we present evidence in humans that specifically implicates the thalamus in this interaction. We used functional magnetic resonance imaging to measure brain activity during the performance of an attentional task under different levels of arousal. Activity evoked in the ventrolateral thalamus by the attentional task changed as a function of arousal. The highest level of attention-related thalamic activity is seen under conditions of low arousal (secondary to sleep deprivation) compared with high arousal (sec- ondary to caffeine administration). Other brain regions were also active during the attentional task, but these areas did not change their activity as a function of arousal. Control experiments establish that this pattern of changes in thalamic activity cannot be accounted for by nonspecific effects of arousal on cerebral hemodynamics. We conclude that the thalamus is involved in mediating the interaction of attention and arousal in humans.

Key words: thalamus; arousal; attention; humans; sleep deprivation; $f M R I$
The relationship between arousal and attention is closely related to the functions of consciousness (Posner, 1994; Harth, 1995). Consciousness is affected by circadian changes of arousal (Tassi et al., 1993) and by the selective influence of attention, which restricts awareness to specific stimuli. Parasuraman (Parasuraman and Davies, 1984) distinguishes sustained from selective attention; the first is defined as maintaining attention to a single source of information for an unbroken period of time, whereas the second is defined as attending to one source of information and excluding the others. Both sustained and selective attention are affected by the level of arousal (Das et al., 1994).

The link between attention and arousal is related to their mutual dependency and complementary behavioral expression (Lynn, 1966). A correlation between performance of attentional tasks and level of arousal has been reported (Babkoff et al., 1991). However, arousal and attention are related in a nonlinear manner: attentional performance improves with a moderate increase of arousal but drops dramatically when a state of high excitement is reached (Easterbrook, 1959). On the other hand, sustained attention reduces arousal and induces drowsiness (Babkoff et al., 1991).

Arousal and attention rely on distinct anatomical systems (Luria, 1973): the system for arousal is mainly subcortical, and the one for attention is mainly cortical. However, the two systems share an important anatomical substrate represented by the thalamus. The relay and reticular neurons of the thalamus exhibit, in animals, a profound change in discharge activity when the brain passes from waking (sustained tonic firing) to sleep onset (bursting mode) (McCormick and Feeser, 1990; Steriade et al., 1993). The relay cells, which receive multi-modal sensory signals, may

Received April 6, 1998; revised Aug. 14, 1998; accepted Aug. 19, 1998.

This work was supported by grants from the Wellcome Trust. C.M.P. holds a Marie Curie Fellowship. We thank Dr. Ivan Toni for helpful suggestions and discussion.

Correspondence should be addressed to Dr. Chiara Maria Portas, Wellcome Department of Cognitive Neurology, Institute of Neurology, 12 Queen Square, WC1N 3BG, London, United Kingdom.

Copyright (C) 1998 Society for Neuroscience $\quad 0270-6474 / 98 / 188979-11 \$ 05.00 / 0$ enhance processing in the cortical areas to which they project (Crick, 1984); in other words, they may modulate the cortical expression of attention (La Berge and Brown, 1989; La Berge et al., 1992; Olshausen et al., 1993; Newman, 1995; Frith and Friston, 1996). It has been suggested recently that in humans the pulvinar and the mediodorsal nuclei of the thalamus represent the targets of a prefrontal top-down (voluntary) modulation of attention (La Berge and Buchsbaum, 1990; La Berge, 1995).

In view of the above considerations, we hypothesize that the degree of thalamic activation [as measured by blood oxygenation level dependent (BOLD) magnetic resonance imaging (MRI)] during an attentional task will differ between conditions of low and high arousal.

A recent study by Coull et al. (1997) has shown evidence in humans consistent with the idea that the thalamus is involved in the interaction between arousal and cognitive performance. They showed that clonidine decreases arousal, impairs cognitive performance (Coull et al., 1995), and decreases the regional cerebral blood flow $(\mathrm{CBF})$ in the thalamus; however, this decrease is reversed during the performance of a cognitive task (Coull et al., 1997).

In this study we sought to clarify the functional relationship between arousal and attention by analyzing attention-related brain activation during different levels of arousal. In particular we attempted to clarify the role of the thalamus in the interaction between arousal and attention.

\section{MATERIALS AND METHODS}

\section{Subjects}

In total eight right-handed, drug-free, healthy subjects (four males and four females), aged 25-38 years, with no history of psychiatric or neurological disorders, were selected for the experiment. Subjects were asked to report about their sleep-wake cycle habits and their daily caffeine intake. We excluded subjects who abused alcohol, had sleep problems (either insomnia or hypersomnia), did not drink coffee at all, or drank four or more cups of coffee (or equivalent caffeinated beverages) per day. Subjects were also required to abstain from caffeine-containing beverages for $48 \mathrm{hr}$ before starting the first experimental session and for the 
duration of the study. All volunteers gave written, informed consent, and the study was approved by the hospital ethics committee.

Subjects were in two groups: "experimental subjects" (six) and "control subjects" (two).

\section{Experimental design: sessions}

The experimental subjects were scanned on 3 consecutive d, with each day/session associated with a different state of arousal. The control subjects were also scanned on 3 consecutive d but under conditions of normal arousal only.

Modulation of the state of arousal in the experimental subjects. Arousal was modulated using caffeine and sleep deprivation. On day 1 subjects were scanned in a state of normal arousal, defined as being awake after a normal night of sleep. On day 2 a state of high arousal was induced by administration of caffeine as described in previous studies (ZwyghuizenDoorenbos et al., 1990; Rosenthal et al., 1991b). Because caffeine may cause unwanted cardiovascular and diuretic effects and may also decrease whole brain CBF (Cameron et al., 1990), we used a dose (5 mg/ $\mathrm{kg}$, p.o.) shown to be effective in increasing the level of arousal without producing significant side effects (Bruce et al., 1986). Peak plasma concentrations are reached in $60 \mathrm{~min}$ and remain constant up to $150 \mathrm{~min}$ (Bruce et al., 1986), so functional magnetic resonance imaging (fMRI) scans were acquired within this time window.

On day 3 subjects were scanned in a state of low arousal induced by sleep deprivation. This experimental manipulation is based on the demonstration that total sleep loss causes a significant decrease of arousal (Babkoff et al., 1991). However, because sleep deprivation may increase stress, affect biological circadian rhythms (Wilkinson, 1965), and produce a reorganization of regional cerebral metabolic activity (Wu et al., 1991), we used a very "gentle" protocol to lessen discomfort for the subjects and minimize possible side effects (Brendel et al., 1990). Subjects were sleep-deprived under continuous supervision for a short period $(24 \mathrm{hr})$ in a quiet environment and in conditions of moderate light to avoid the resetting of the circadian pacemaker (Klerman et al., 1996).

The order of the scanning sessions was constant for all experimental subjects (normal arousal on day 1, high arousal on day 2, and low arousal on day 3). Because the effects of sleep deprivation last for several days (Rosenthal et al., 1991a), it was not appropriate to sleep-deprive a subject on day 1 or 2 and then move to another experimental manipulation on day 2 or 3 . We considered using a counterbalanced design with sessions separated by several days, but decided that the advantages gained by counterbalancing of order would be lost by the likely changes in the subjects' state and the difficulty of achieving compliance with the requirement to abstain from caffeine-containing beverages across such a long period. Instead we examined the possibility of systematic changes over time by using a small number of control subjects (see Control experiments for nonspecific effects).

Assessment of arousal. Subjective ratings of arousal were measured over the $3 \mathrm{~d}$ to verify the achievement of the desired state. Before and after each scanning session subjects were asked to evaluate their state using a modified version of the Stanford Sleepiness Scale Assessment (Babkoff et al., 1991) and to express it as alert (1 point), awake ( 2 points), drowsy ( 3 points), or almost asleep (4 points). The sum of these two evaluations was used to rate the state as "normal arousal" (score between 4 and 5), "high arousal" (between 2 and 3), and "low arousal" (between 6 and 8).

\section{Experimental design: conditions}

In each scanning session functional images were acquired in $\sim 22 \mathrm{~min}$ during 10 repetitions of the following four conditions: passive viewing of a reversing black and white checkerboard ("checkerboard"), fixation of a blank screen ("blank screen"), attention and response to a visual stimulus ("attentional task"), and passive viewing of a visual stimulus ("passive viewing") (Fig. 1 $a-d$ ). The order of the four conditions was kept constant in all subjects. By repeating the four conditions in a fixed order we insured maximum change per condition across time. This variation is optimum for detecting differences in BOLD signal, minimizes differences between subjects, and also reduces the likelihood of changes in level of arousal within-session as might occur with long periods of "passive viewing" (which would tend to reduce arousal) or long periods of attentional performance (which would tend to affect performance results). It is known, for example, that the effects of sleep deprivation on cognitive performance depend on the duration of the task (Wilkinson, 1968).

Attentional task. Subjects were trained to respond, while lying in the scanner, to a visual target (the number 7) that appeared for $600 \mathrm{msec}$ at an interval varying between 600 and $2400 \mathrm{msec}$ at one of four preset
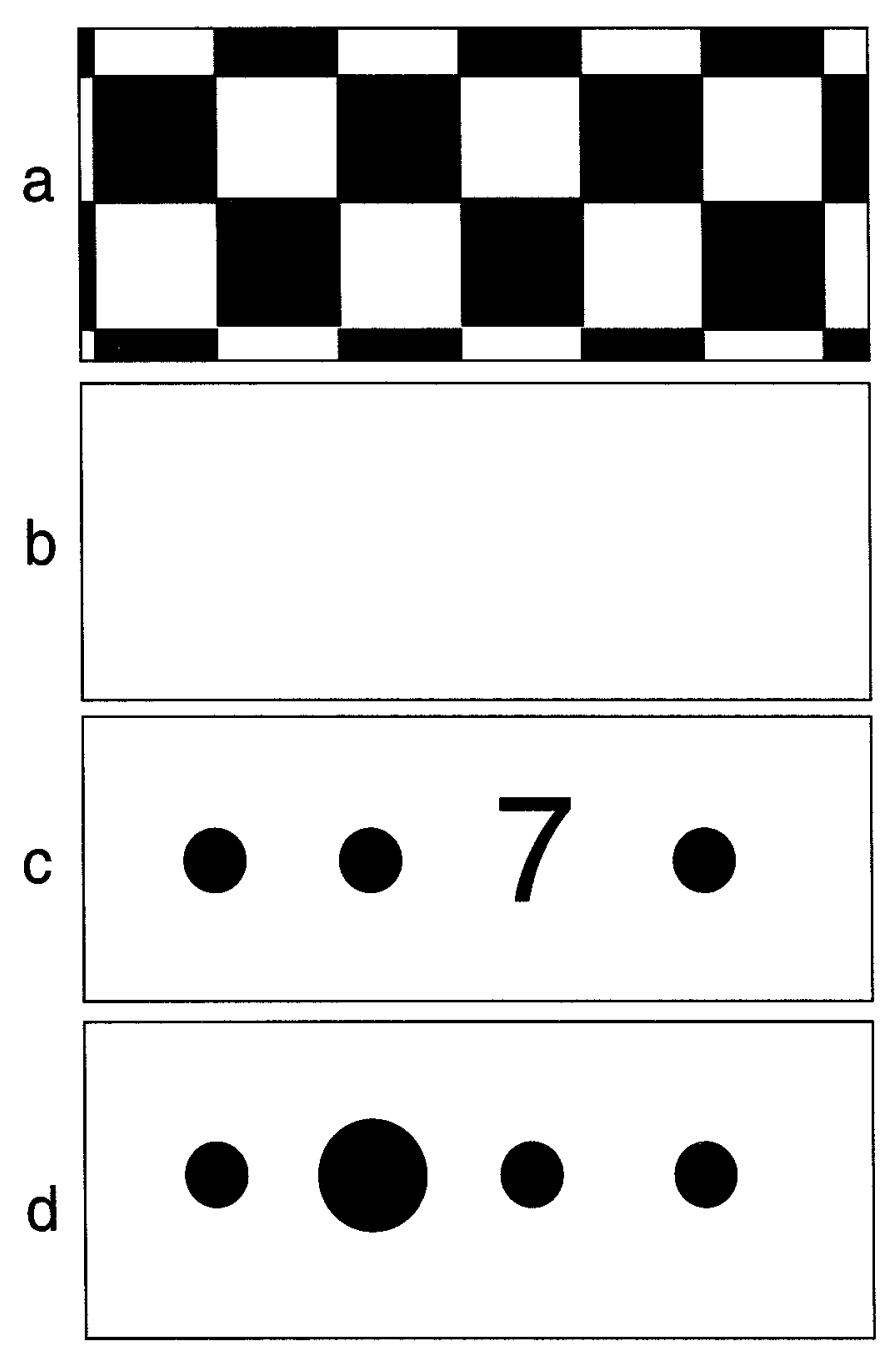

Figure 1. Schematic representation of the four conditions used in the experimental paradigm. $a$, The passive viewing of a reversing black and white checkerboard; $b$, fixation of a blank screen, representing the contrast condition for the checkerboard; $c$, the attentional condition in which subjects had to respond to a target (7) appearing randomly at one of four positions on the screen marked by flashing dots; $d$, the contrast condition for the attentional task in which only four flashing dots appeared on the screen, one of which enlarged randomly.

positions (Fig. 1c). The four positions were marked with large flashing dots and were spatially organized to minimize eye movements (requiring foveal or parafoveal fixation). During the task several numeric distracters appeared randomly in place of the target. Subjects were asked to respond to the target and its location by pressing, with the fingers of their right hand, one of four buttons placed on a key pad and corresponding to the four positions on the screen. In the condition used as baseline for the attentional task (passive viewing), four flashing dots appeared on the screen, and one of them was randomly enlarged compared with the others (Fig. $1 d$ ).

Performance of the attentional task was estimated in each subject in the three experimental sessions (normal, high, and low arousal in the experimental subjects and $3 \mathrm{~d}$ of normal arousal in the controls) in terms of mean reaction time (computed only for correct target identification) and total number of mistakes (false positive and false negative in target identification over 140 trials per session). A repeated-measures ANOVA was used to test differences among groups (group performance of the experimental subjects in normal, high, and low arousal).

The task had a short duration (32 sec per repetition) to allow the subjects to maintain a consistent level of performance during different levels of arousal. In fact the effects of sleep loss on cognitive performance is normally seen with longer and more demanding tasks (Heslegrave and 
Angus, 1985). Matching performance across arousal states was important in this study to avoid confounding changes in evoked activity attributable to arousal or attention with those attributable to changes in performance.

Each fMRI session started approximately at the same time (between 9:00 and 10:30 A.M.) to minimize the effect of circadian cycle on cognitive performance (Babkoff et al., 1991).

\section{Control experiments for nonspecific effects}

Because this study examines specifically the interaction between arousal and attention, it was necessary to control for (1) nonspecific hemodynamic effects secondary to changes in arousal (or caused by caffeine or sleep deprivation per se) and (2) nonspecific hemodynamic effects secondary to time (effects that may be produced by the noncounterbalanced order of the sessions, by day-to-day variability in BOLD signal, or by task habituation).

To identify nonspecific hemodynamic effects attributable to changes in arousal, caffeine, or sleep deprivation, we used passive checkerboard stimulation. Such a visual stimulus evokes a highly reproducible pattern of activation unrelated to incidental cognitive processes. If changes in arousal across sessions produced nonspecific changes in cerebral hemodynamics, we expected to see changes in the activity evoked by such a stimulus. It is particularly important to account for hemodynamic effects caused by caffeine or sleep deprivation because sleep deprivation has been shown to decrease regional cerebral metabolic activity in the thalamus (Wu et al., 1991) and caffeine affects global CBF without modifying regional CBF (Cameron, 1990).

To control for time effects, two control subjects were scanned on 3 consecutive $d$ in a state of normal arousal during the same four conditions used for the experimental subjects. There was no manipulation of arousal in control subjects, so we expected that any nonspecific time effects would be manifest as condition-specific changes in pattern of activity over time.

\section{Image acquisition}

In each subject, anatomical (T1 weighted) and functional images (T2*) were acquired with a 2 Tesla Magnetom VISION MRI scanner (Siemens, Erlangen, Germany). Functional images consisted of contiguous multislice gradient-echo, echo-planar $\mathrm{T} 2 *$ weighted image volumes obtained with BOLD contrast using an axial slice orientation with an echo time of $40 \mathrm{msec}$. BOLD fMRI uses deoxyhemoglobin (which is more paramagnetic than oxyhemoglobin) as an endogenous contrast agent. Any modification of the brain state that creates an imbalance between oxygen uptake and blood flow in a given brain region will cause a change of deoxyhemoglobin level and related MRI signal in the vessels of that brain region (Turner, 1992).

The volume acquired covered the whole brain (64 slices, voxel size $3 \times$ $3 \times 3 \mathrm{~mm}$ ); the field of view was $192 \mathrm{~mm}$. The effective repetition time between volumes was $6.4 \mathrm{sec}$.

\section{Data analysis}

Imaging data were analyzed as a series of single cases rather than as group so that subtle effects would not be swamped by individual differences. Group analysis of imaging data depends on "normalization" of individual brain images into a standard space. Such normalization can never fully take account of individual differences in functional anatomy and will be particularly problematic when the focus of interest is a small complex structure such as the thalamus (see below).

Processing of the data. The 200 functional volumes acquired in each subject (per session) were realigned, spatially normalized to the stereotactic space of Talairach and Tournoux (1988), and smoothed (spatially filtered) using a $6 \mathrm{~mm}$ Gaussian kernel (Friston et al., 1996). To maximize the possibility of identifying small anatomical regions, the structural image of each subject was co-registered to his/her own functional images so that the areas of activation superimposed to the suitable anatomical structures.

Global changes in BOLD signal were removed by proportional scaling (Holmes et al., 1997), and values were mean-adjusted. After the appropriate experimental design matrix was specified, the covariates of interest (condition-specific effects) were estimated according to the General Linear Model at each and every voxel (Friston et al., 1995), and lowfrequency fluctuations were modeled as covariates of noninterest. The best square-fit of the adjusted data to the modeled experimental conditions represents the parameter estimates (Buechel and Friston, 1997).

To test the hypothesis about regional condition-specific effects, the estimates were compared using linear contrast.
Statistical inference. Significant hemodynamic changes for each contrast were assessed using $t$ statistics on a voxel-by-voxel basis (on $\sim 200,000$ voxels per brain volume). The set of $t$ values thus obtained constituted a statistical parametric map (SPM) ( $t$ ) (Friston et al., 1995). This map was transformed into SPM $(Z)$. Resultant areas of activation were characterized in terms of their peak heights. We report activations above a threshold corresponding to $p<0.001$ uncorrected for multiple comparisons $(Z>3.09)$ for the area defined in our hypothesis (the thalamus). Outside this area we made a correction for multiple comparisons across the whole brain volume examined and report only areas of activity above a threshold corresponding to $p<0.05$ corrected $(Z>4.5)$. This correction is achieved with standard procedures that correct for the multiplicity of voxels and the spatial correlation among them on the basis of the theory of the random Gaussian fields (Friston et al., 1994).

Functional contrasts relevant to the experimental design. To measure hemodynamic changes in the brain it is necessary to match pairs of activation/baseline conditions. The activation task engages the cognitive component of interest, whereas the baseline condition does not engage that component. In this way the neural correlates of the cognitive component of interest are revealed by subtracting the activity during the baseline condition from that of the activation condition. This simple type of analysis is referred as subtraction analysis (Price et al., 1997).

In this study the pair attentional task/passive viewing was used to identify attention-dependent hemodynamic effects, whereas the pair checkerboard/blank screen was used as a control experiment to characterize attention-independent hemodynamic effects.

To identify, in each subject, the changes in brain activation related to attention, to arousal, and in particular to the interaction between arousal and attention, we used conjunction and interaction analyses in addition to the subtraction analysis. These two types of analyses represent an extension of the simple subtraction analysis. Conjunction analyses reveal the common activation differences between two or more pairs of conditions (e.g., the pair attentional task/passive viewing during low arousal and the same pair during high arousal), whereas interaction analyses identify the neural activity that is specific to one or other condition pair (for an extensive review of these types of analysis, see Price et al., 1997). The experimental design in this study included three sessions representing a different (experimental subjects) or the same (control subjects) level of arousal and four conditions; therefore, two variables have to be tested (session and condition-related brain activation). This constitutes a factorial design. Interaction analyses allow us to measure explicitly the effect that one variable (session $=$ e.g., low arousal) has on the expression of the other variable (condition = e.g., attention).

We present here three types of contrasts used in this study to identify the brain activations relevant to the experimental design: (1) conditionspecific effects within sessions (simple subtraction analysis); (2) condition-specific effects between sessions (conjunction and interaction analysis); (3) nonspecific hemodynamic changes between sessions (conjunction and interaction analysis). Condition-specific effects within sessions included attention-dependent effects ("attentional task" versus its baseline "passive viewing" in low, high, and normal arousal); attentionindependent effects ("checkerboard" stimulation versus "blank screen" in normal, high, and low arousal). Condition-specific effects between sessions included effects of attention on the level of arousal ("attentional task" versus "passive viewing" in normal compared with high or low arousal); effects of visual stimulation dependent on the level of arousal or caffeine or sleep deprivation ("checkerboard" stimulation versus "blank screen" in normal, compared with high or low arousal). Nonspecific hemodynamic changes between sessions (normal arousal only) were assessed in the two control subjects to identify possible hemodynamic changes attributable to any time confound " "attentional task" versus "passive viewing" and "checkerboard" stimulation versus "blank screen" in day 1 , compared with day 2 and day 3 ).

\section{RESULTS}

\section{Arousal assessment}

The subjective evaluation of the state of arousal [Stanford Sleepiness Scale Assessment (Babkoff et al., 1991)] showed that all subjects and controls gave an estimate consistent with the experimental manipulation (mean group \pm SD was $4.4 \pm 0.54$ in normal arousal, $2.6 \pm 0.89$ in high arousal, $6.6 \pm 0.89$ in low arousal). 
Figure 2. Activation in the left and right thalamic regions (centered in the inset) in each subject in the contrast attentional task versus passive viewing in normal, high, and low arousal. Note the greater activation when the test is performed in a state of low arousal. In each subject the areas of activation are superimposed on the correspondent structural images (which are co-registered to the functional ones). This procedure permits greater sensitivity in identifying anatomical structures because there is no need to account for variations in normal anatomy between subjects. The apparent activations in two subjects in the ventricles are probably artifactual. The $Z$-value represents the degree of significance of the activations (see Materials and Methods).

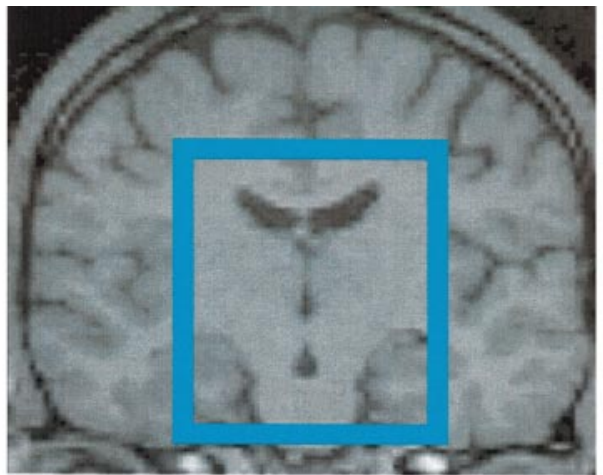

\section{low arousal

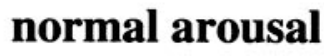 \\ high arousal}

subject 1
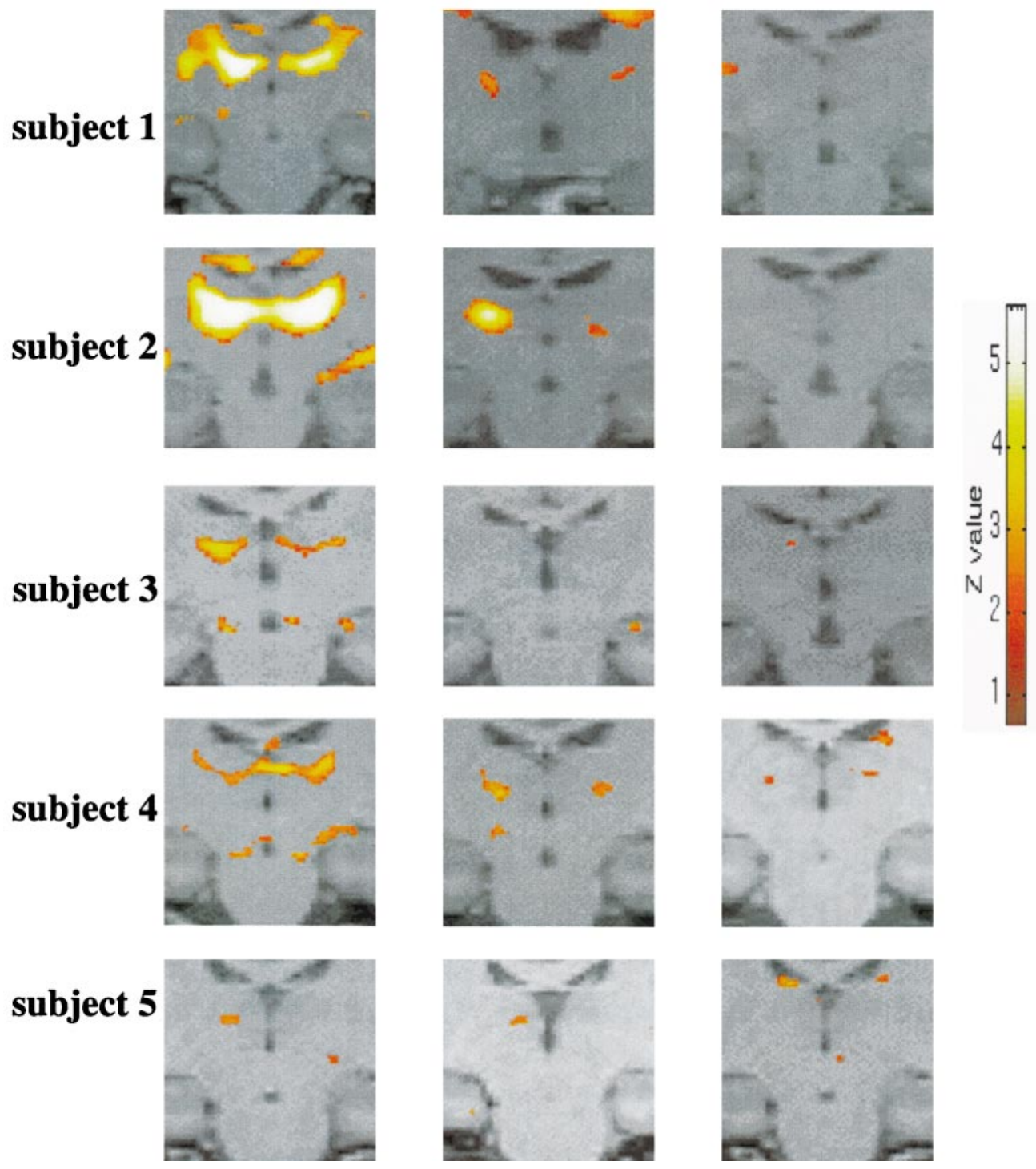

\section{Attentional performance}

Attentional performance was measured as mean reaction time and total number of mistakes in target identification over 140 trials. In the experimental subjects the group mean reaction time milliseconds \pm SEM was $444.90 \pm 15.22$ in normal arousal, $445.48 \pm 23.22$ in high arousal, and $456.98 \pm 14.23$ in low arousal. The group mean number of mistakes (including false negatives and false positives) was $27.6 \pm 9.04$ in normal arousal, $25.6 \pm 8.05$ in high arousal, and $37 \pm 11.3$ in low arousal. A repeatedmeasures ANOVA showed that there were no significant differences between the three sessions $(p>0.1)$.

In the two control subjects the attentional task was always performed in a state of normal arousal with highly consistent results (data not shown). 
Table 1. Maxima and coordinates of attention-related brain activation

\begin{tabular}{|c|c|c|c|c|c|c|c|c|}
\hline & \multicolumn{4}{|c|}{ Left hemisphere } & \multicolumn{4}{|c|}{ Right hemisphere } \\
\hline & \multicolumn{3}{|c|}{ Coordinates } & \multirow[b]{2}{*}{$Z$-score } & \multicolumn{3}{|c|}{ Coordinates } & \multirow[b]{2}{*}{$Z$-score } \\
\hline & $x$ & $y$ & $z$ & & $x$ & $y$ & $z$ & \\
\hline \multicolumn{9}{|l|}{$\overline{\mathrm{sP}}$} \\
\hline Subject 1 & -24 & -66 & 56 & 9.58 & 26 & -66 & 36 & 6.46 \\
\hline Subject 2 & -30 & -62 & 44 & 11.54 & 34 & -62 & 50 & 11.34 \\
\hline Subject 3 & -26 & -64 & 64 & 12.66 & 28 & -66 & 60 & 11.08 \\
\hline Subject 4 & -30 & -60 & 54 & 11.33 & 20 & -74 & 52 & 7.12 \\
\hline Subject 5 & -28 & -68 & 40 & 5.67 & 36 & -68 & 28 & 10.82 \\
\hline Control 1 & -28 & -68 & 46 & 5.56 & 30 & -50 & 42 & 6.53 \\
\hline Control 2 & -22 & -58 & 46 & 14.55 & 28 & -66 & 54 & 9.69 \\
\hline \multicolumn{9}{|l|}{ iP } \\
\hline Subject 1 & -42 & -34 & 38 & 14.12 & 44 & -26 & 40 & 11.65 \\
\hline Subject 2 & -36 & -44 & 34 & 8.29 & & & & $<4.5$ \\
\hline Subject 3 & -32 & -28 & 58 & 9.55 & & & & $<4.5$ \\
\hline Subject 4 & -28 & -46 & 40 & 12.34 & 30 & -46 & 44 & 9.48 \\
\hline Subject 5 & -38 & -34 & 64 & 9.29 & 40 & -30 & 64 & 10.21 \\
\hline Control 1 & -44 & -28 & 44 & 8.87 & 26 & 16 & 56 & 4.84 \\
\hline Control 2 & -40 & -34 & 62 & 9.53 & & & & $<4.5$ \\
\hline \multicolumn{9}{|l|}{$\mathrm{pF}$} \\
\hline Subject 1 & -40 & 44 & 12 & 6.78 & 38 & 38 & 16 & 7.24 \\
\hline Subject 2 & -34 & 46 & 26 & 7.53 & 30 & 42 & 20 & 8.21 \\
\hline Subject 3 & -44 & 34 & 26 & 9.67 & 34 & 30 & 36 & 8.21 \\
\hline Subject 4 & -30 & 44 & 10 & 9.73 & 42 & 38 & 24 & 5.81 \\
\hline Subject 5 & & & & $<4.5$ & & & & $<4.5$ \\
\hline Control 1 & & & & $<4.5$ & 38 & 48 & 26 & 6.22 \\
\hline Control 2 & -22 & 40 & 32 & 6.53 & 38 & 38 & 34 & 8.87 \\
\hline \multicolumn{9}{|l|}{ pM } \\
\hline Subject 1 & -26 & -6 & 48 & 13.64 & 32 & -8 & 52 & 9.36 \\
\hline Subject 2 & -36 & 6 & 30 & 10.87 & 34 & 6 & 28 & 10.97 \\
\hline Subject 3 & -32 & -10 & 64 & 11.67 & 40 & -8 & 66 & 11.06 \\
\hline Subject 4 & -38 & -4 & 42 & 12.07 & 36 & -4 & 40 & 10.29 \\
\hline Subject 5 & -28 & -6 & 48 & 13.77 & 30 & -6 & 54 & 12.02 \\
\hline Control 1 & -42 & -2 & 34 & 6.26 & 48 & 6 & 34 & 8.99 \\
\hline Control 2 & -48 & -2 & 36 & 14.15 & 48 & -2 & 38 & 13.82 \\
\hline \multicolumn{9}{|l|}{$\mathrm{aC}$} \\
\hline Subject 1 & -2 & 8 & 44 & 11.80 & 6 & 14 & 38 & 5.98 \\
\hline Subject 2 & -8 & 12 & 38 & 9.77 & 8 & 8 & 44 & 7.65 \\
\hline Subject 3 & 0 & -2 & 48 & 9.77 & 0 & -2 & 48 & 9.77 \\
\hline Subject 4 & -8 & 12 & 38 & 10.98 & 10 & 12 & 38 & 6.99 \\
\hline Subject 5 & & & & $<4.5$ & & & & $<4.5$ \\
\hline Control 1 & 4 & -2 & 46 & 7.93 & 8 & 4 & 46 & 9.76 \\
\hline Control 2 & -4 & 6 & 34 & 11.37 & 8 & 12 & 32 & 14.16 \\
\hline \multicolumn{9}{|l|}{$\mathrm{O}$} \\
\hline Subject 1 & -20 & -96 & 10 & 8.01 & 30 & -92 & -2 & 5.06 \\
\hline Subject 2 & -36 & -74 & -14 & 8.80 & 24 & -82 & -8 & 8.27 \\
\hline Subject 3 & -28 & -96 & -8 & 10.33 & 34 & -96 & -6 & 10.31 \\
\hline Subject 4 & -32 & -92 & 14 & 7.78 & 28 & -96 & 0 & 4.88 \\
\hline Subject 5 & -28 & -96 & 6 & 11.59 & 16 & -98 & 6 & 6.24 \\
\hline Control 1 & -22 & -90 & -12 & 4.81 & 40 & -90 & -4 & 7.30 \\
\hline Control 2 & -28 & -96 & -6 & 14.35 & 36 & -96 & -8 & 14.82 \\
\hline \multicolumn{9}{|l|}{$\mathrm{Ce}$} \\
\hline Subject 1 & -26 & -62 & -28 & 8.13 & 40 & -58 & -30 & 11.84 \\
\hline Subject 2 & -30 & -68 & -46 & 8.04 & 14 & -70 & -46 & 9.51 \\
\hline Subject 3 & -26 & -58 & -20 & 7.0 & 18 & -52 & -16 & 13.19 \\
\hline Subject 4 & -26 & -64 & -22 & 7.42 & 32 & -54 & -28 & 9.37 \\
\hline Subject 5 & -28 & -54 & -28 & 6.89 & 30 & -52 & -34 & 6.92 \\
\hline Control 1 & -28 & -56 & -22 & 7.49 & 22 & -64 & -22 & 9.08 \\
\hline Control 2 & -30 & -58 & -24 & 12.05 & 22 & -70 & -26 & 10.07 \\
\hline
\end{tabular}

Talairach coordinates $(x, y, z)$ and maxima ( $Z$-score) relative to the commonalities in brain activation (conjunction analysis) in the contrast "attentional task" versus "passive viewing" in normal, high, and low arousal (for the experimental subjects) and in three sessions of normal arousal (for the control subjects). sP, Superior parietal gyrus; iP, intraparietal sulcus/postcentral gyrus; $\mathrm{pF}$, prefrontal cortex; $\mathrm{pM}$, premotor cortex; aC, anterior cingulate; $\mathrm{O}$, occipital cortex; $\mathrm{Ce}$, cerebellum. 


\section{left hemisphere parasagittal view}

\section{both hemispheres axial view}

\section{right hemisphere parasagittal view}
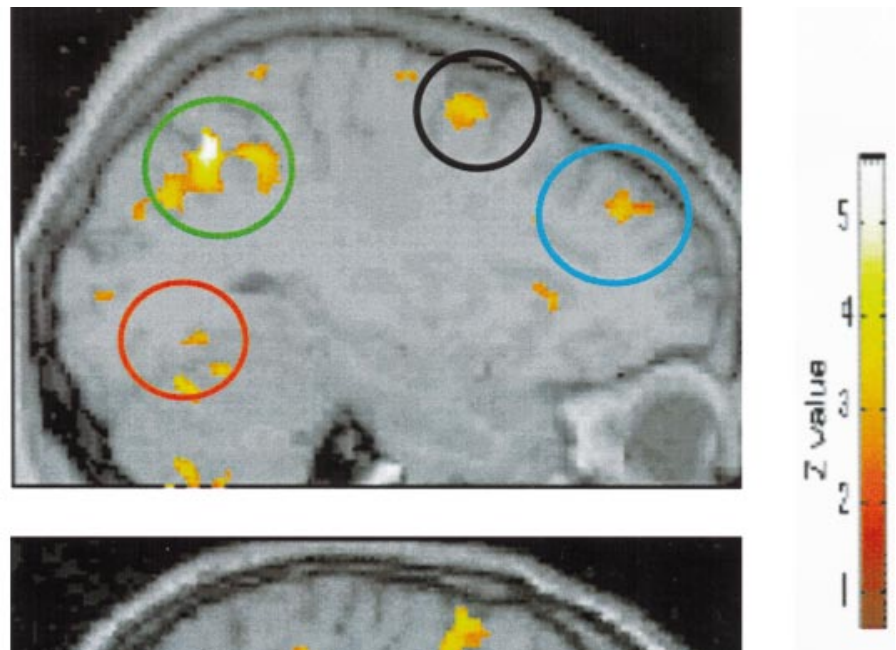

Figure 3. An example (in one experimental subject) of the common pattern of bilateral activation in the parietal, prefrontal, premotor, anterior cingulate, and occipital cortices, and cerebellum in the contrast attentional task versus passive viewing in normal, high, and low arousal (conjunction analysis). The $Z$-value represents the degree of significance of the activations (see Materials and Methods).

\section{FROM FRONT TO BACK:}

- prefrontal cortex (ant. part of the middle frontal gyrus)

- premotor cortex (inf. precentral sulcus)

- ant. part of the cingulate sulcus

- sup. parietal gyrus

- fusiform gyrus

- cerebellum

\section{Imaging data}

In one experimental subject imaging data were not analyzed because of failure of the realignment procedure (the subject produced head movements in excess of $20 \mathrm{~mm}$ ). Consequently data are presented for five experimental subjects only and for two control subjects.

Results are reported for significant effects occurring in at least four out of five subjects or in both the controls (unless otherwise specified).

Attentional task versus passive viewing in normal, high, and low arousal

In all five subjects the performance of the attention-demanding task during a state of low arousal produced a greater activation of 


\begin{tabular}{|c|c|c|c|c|c|c|c|c|}
\hline & \multicolumn{4}{|c|}{ Left hemisphere } & \multicolumn{4}{|c|}{ Right hemisphere } \\
\hline & \multicolumn{3}{|c|}{ Coordinates } & \multirow{2}{*}{$\begin{array}{l}Z \text { - } \\
\text { score }\end{array}$} & \multicolumn{3}{|c|}{ Coordinates } & \multirow{2}{*}{$\begin{array}{l}Z- \\
\text { score }\end{array}$} \\
\hline & $x$ & $y$ & $z$ & & $x$ & $y$ & $z$ & \\
\hline Subject 1 & -8 & -76 & -14 & 14.37 & 10 & -78 & -8 & 14.43 \\
\hline Subject 2 & -26 & -70 & -14 & 13.87 & 4 & -92 & 8 & 14.19 \\
\hline Subject 3 & -14 & -80 & -12 & 12.49 & 24 & -76 & 20 & 13.24 \\
\hline Subject 4 & -18 & -96 & 8 & 12.44 & 12 & -82 & -6 & 12.70 \\
\hline Subject 5 & -6 & -70 & -4 & 13.71 & 20 & -80 & 20 & 14.18 \\
\hline Control 1 & -6 & -92 & -2 & 12.53 & 12 & -92 & 0 & 12.57 \\
\hline Control 2 & -6 & -78 & -6 & 15.16 & 0 & -92 & 8 & 15.55 \\
\hline Mean & -6 & -85 & -4 & & 6 & -92 & 4 & \\
\hline
\end{tabular}

Talairach coordinates $(x, y, z)$ and maxima ( $Z$-score) relative to the commonalities in brain activation (conjunction analysis) in the contrast "checkerboard" versus "blank screen" in normal, high, and low arousal (for the experimental subjects) and in three sessions of normal arousal (for the control subjects).

Table 3. Maxima and coordinates of thalamic activation

\begin{tabular}{|c|c|c|c|c|c|c|c|c|}
\hline & \multicolumn{4}{|c|}{ Left hemisphere } & \multicolumn{4}{|c|}{ Right hemisphere } \\
\hline & \multicolumn{3}{|c|}{ Coordinates } & \multirow[b]{2}{*}{$Z$-score } & \multicolumn{3}{|c|}{ Coordinates } & \multirow[b]{2}{*}{$Z$-score } \\
\hline & $x$ & $y$ & $z$ & & $x$ & $y$ & $z$ & \\
\hline Subject 1 & -12 & -14 & 6 & 4.03 & 6 & -10 & 4 & 5.23 \\
\hline Subject 2 & -8 & -14 & 8 & 7.42 & 14 & -18 & 8 & 7.64 \\
\hline Subject 3 & -16 & -12 & 12 & 3.14 & 22 & -10 & 14 & 3.31 \\
\hline Subject 4 & -20 & -16 & 16 & 3.71 & 18 & -12 & 14 & 4.35 \\
\hline Subject 5 & & & & $<3.09$ & & & & $<3.09$ \\
\hline Mean & -14 & -14 & 10 & & 15 & -12 & 10 & \\
\hline
\end{tabular}

Differential activation in the contrast "attentional task" versus "passive viewing" in low arousal versus high arousal (interaction analysis). Talairach coordinates $(x, y, z)$ and maxima ( $Z$-score) are expressed for each subject and coordinates only for group mean.

the ventrolateral part of the thalamus (unilateral in subject 5) compared with the activation in normal or high arousal (withinsession subtraction analyses) (Fig. 2). This change of activity was highly specific for the thalamus. Other cortical areas, also active during the attention task, did not show consistent changes in activity during low or high arousal across subjects (see interaction between attention and arousal). The common areas of brain activation produced by the attentional task across the three sessions are summarized in Table 1 for each subject (betweensessions conjunction analyses). Parietal activation was observed in two areas: the intraparietal sulcus and the superior parietal gyrus [Brodmann area (BA) 7, 40]. Prefrontal activation was localized mainly to the most anterior part of the middle frontal gyrus (BA 46); premotor activation included the superior precentral sulcus and the most anterior part of the precentral gyrus (BA 6). The anterior part of the cingulate sulcus and gyrus (BA 32), part of the middle occipital gyrus and (in two subjects) of the fusiform gyrus (BA 17,18), and a small area of the cerebellum were also activated in all subjects. Figure 3 illustrates these areas of activation in one subject.

\section{Checkerboard versus blank screen in normal, high, and low arousal}

The contrast checkerboard versus blank screen produced in each subject significant activation of the striate (primary visual cortex
V1) and prestriate occipital cortex (V2, V3), often more evident in the lingual gyrus. In two subjects the extrastriate cortical area that selectively responds to motion (V5) was also activated (within-session subtraction analyses). This pattern of activation was highly consistent across the $3 \mathrm{~d}$ in normal, high, and low arousal. Table 2 shows the highly significant $Z$-score $(Z>12)$ related to the common activation across the three sessions (normal, high, and low arousal) (between-sessions conjunction analyses).

\section{Interaction between attention and arousal}

The hemodynamic changes in the contrast attentional task versus passive viewing during low arousal as compared with high or normal arousal confirmed the higher activation of the ventrolateral part of the thalamus (between-sessions interaction analyses) (Table 3, Fig. 4). This activation was significant in four subjects when comparing low versus high arousal and in three subjects when comparing low versus normal arousal. This suggests that the thalamus is least activated when attentional performance is required in a state of high arousal (between-sessions interaction analyses; data not shown). The cortical areas also active during the attentional task did not show consistent changes in activity across subjects when comparing low with normal or high arousal (between-sessions interaction analyses; data not shown).

\section{Interaction between nonattention demanding task (checker board stimulation) and arousal}

In the contrast checkerboard versus blank screen there were no consistent changes in brain activity across subjects as a function of arousal (between-sessions interaction analyses; data not shown).

\section{Single voxel analysis in the thalamus: a group analysis}

The impression given by the series of single-subject analyses was confirmed by performing a group analysis on a single voxel region of interest in the thalamus. Figure 5 shows the changes of the parameter estimates in this thalamic voxel (coordinates $x=-12$, $y=-18, z=8$ ) during attentional task and passive viewing (Fig. 5 , graph on the left) and checkerboard and blank screen (graph on the right). This thalamic voxel was selected because it showed a significant attention-related change of activity in all single-subject analyses under low arousal. It is evident from the figure that the signal is high during the attentional task under any condition of arousal, whereas it is below or around mean values during checkerboard, blank screen, and passive viewing conditions. This suggests that this specific voxel does not respond to any visual stimulation unless attention is required. Furthermore, the attention-related signal reaches its highest levels under low arousal.

The attention-related activation in this voxel (quantified as the difference in mean parameter estimates values between attentional task and passive viewing) is higher under low arousal as compared with the high arousal condition (ANOVA between the three levels of arousal, $p<0.05$, followed by post hoc one-tailed $t$ test, $p<0.05)$.

\section{Attentional task versus passive viewing in the controls (normal arousal)}

In contrast to the change of thalamic activation produced in four of five subjects by the attentional task as a function of arousal, no significant change in thalamic activity was shown in the control subjects between any session [between-sessions interaction anal- 


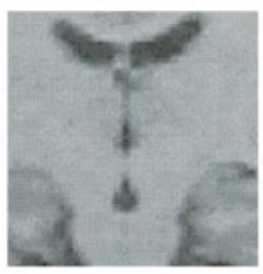

control 1

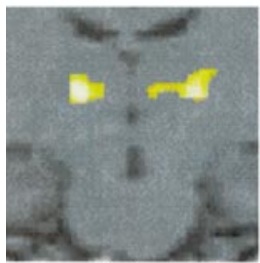

subject 1

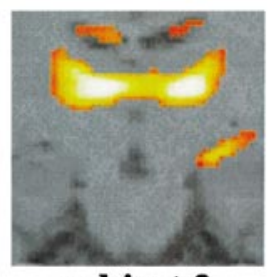

subject 2

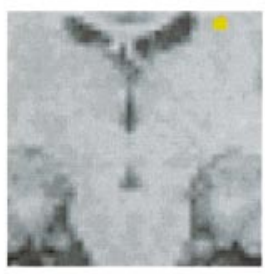

control 2

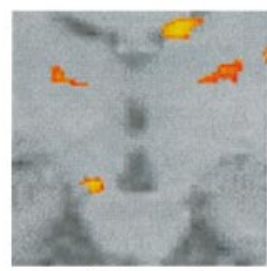

subject 3

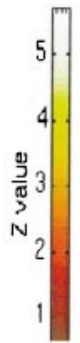

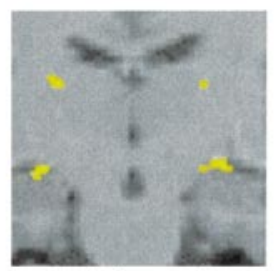

subject 4

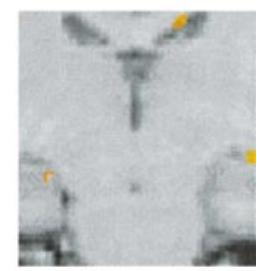

subject 5

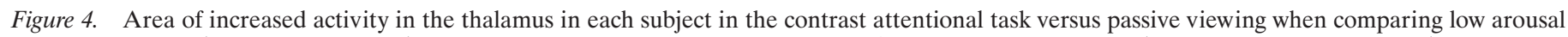
with high arousal (interaction analysis). The $Z$-value represents the degree of significance of the activations (see Materials and Methods).

yses (Fig. 4)]. The attentional task produced a consistent bilateral activation of the parietal, prefrontal, premotor, cingulate, and occipital cortices in analogy to the cortical activation seen in the five experimental subjects [between-sessions conjunction analyses (Table 1)]. This did not change consistently over the three sessions of normal arousal (between-sessions interaction analyses; data not shown).

\section{Checkerboard versus blank screen in the controls (normal arousal)}

The contrast checkerboard versus blank screen produced a significant activation of the striate and prestriate cortex in the two controls comparable to the activation shown in the five subjects [between-sessions conjunction analyses (Table 2)]. This did not change consistently over the three sessions of normal arousal (between-sessions interaction analyses; data not shown).

\section{DISCUSSION}

This study used fMRI in humans to identify the hemodynamic changes related to the performance of an attention-demanding task while changing the level of arousal. We examined the hypothesis that a change of functional activation would occur in the thalamus as an expression of the specific interaction between the attentional and arousal systems. The results suggest several points for discussion.

\section{Arousal assessment and task performance}

All subjects and controls gave a subjective evaluation of the state of arousal consistent with the experimental manipulation. This result validates the protocol used to modulate arousal.

There was no significant change in attentional performance during different levels of arousal. This is not surprising because effects of sleep loss on cognitive performance have been reported only for longer and more demanding tasks (Wilkinson, 1968), and caffeine (at the dose used in this study) does not affect attentional performance (Bruce et al., 1986). This finding is very important because it shows that greater activation of the thalamus during low arousal was not related to a change of task performance but was caused by the specific interaction between attention and arousal. We also considered the possibility that, within each subject, the change of performance may correlate with the degree of activation of the ventrolateral thalamus. Thus we compared the change of activation of each subject with the individual performance score; however, this comparison did not show consistent results (data not shown).

Consequently the modulation of thalamic activity may relate to the effort of maintaining attention during the task (Kahneman, 1973). This possibility is consistent with the observation that the accomplishment of the task during low arousal was associated with a subjective feeling of "mental effort" (four of six subjects spontaneously reported a sense of fatigue and strong effort when performing the attentional task under low arousal condition).

Thalamic activation as an expression of the interaction between arousal and attention

The major finding of this study is that there was a greater change of activity (between attentional task and baseline condition) in the ventrolateral thalamus in a state of low arousal compared with the change of activity shown in a state of normal or high arousal. We suggest that the change of attention-related thalamic activation as a function of arousal directly reflects a different degree of interaction between the arousal and the attention systems under sleep deprivation.

The control experiments show that this greater change of thalamic activity cannot be accounted for by sleep deprivation or low arousal per se because it appears only when attention is required, and it cannot be related to nonspecific hemodynamic effects related to time because there was no significant change of thalamic activity in the control subjects across sessions (normal arousal only). Finally, the modulation of thalamic activation we observed cannot be related to changes in attentional performance because all subjects performed similarly during normal, high, and low arousal.

\section{Physiological interpretation of the attention-related thalamic activation during low arousal}

We speculate that several thalamic nuclei situated in the ventrolateral region are involved in the interaction between arousal and attention. The somatosensory relays, the ventrolateral nucleus (part of the motor thalamus), the centromedian nucleus (linked to 

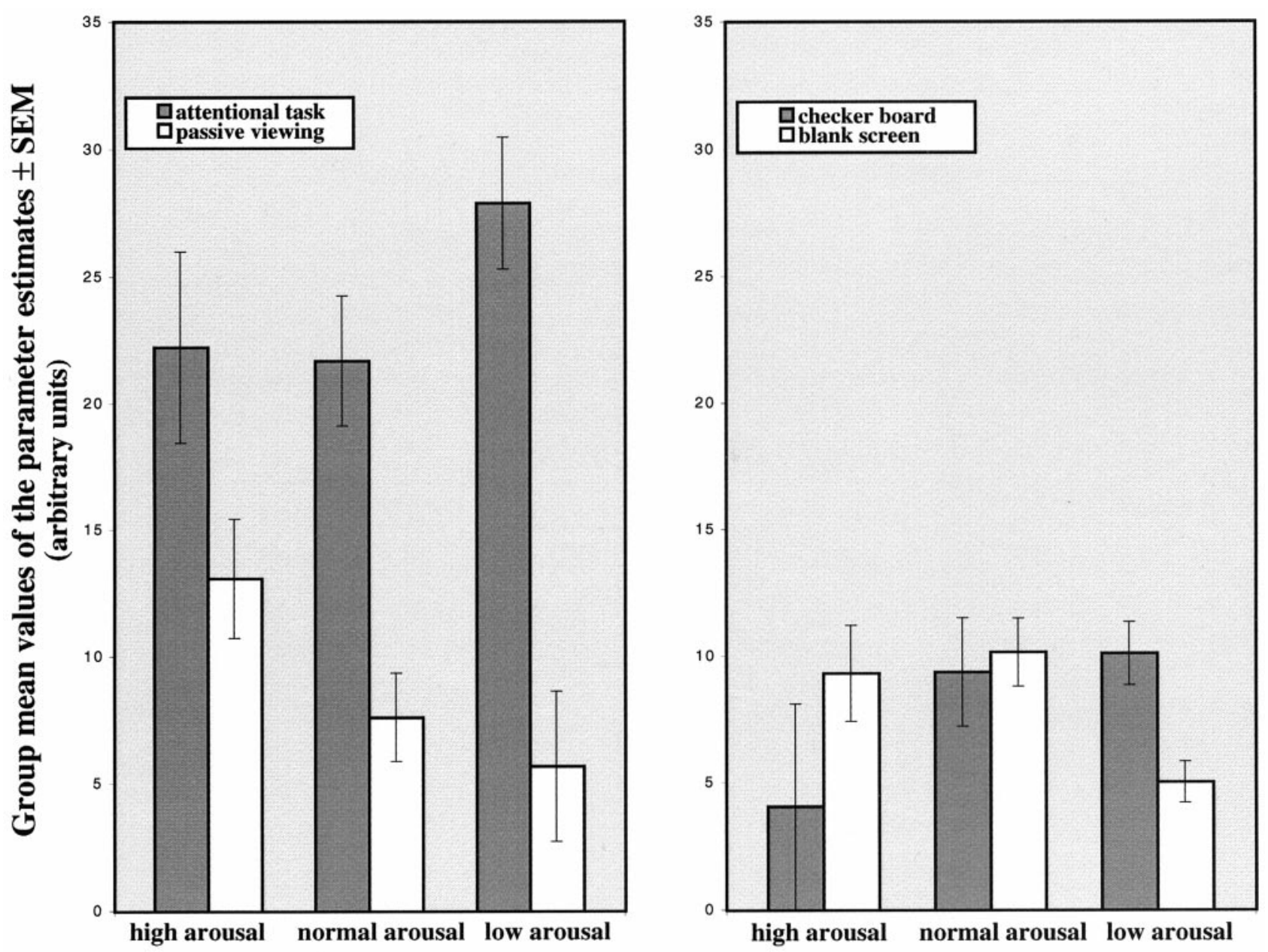

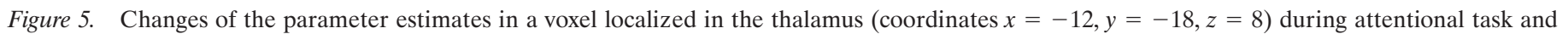

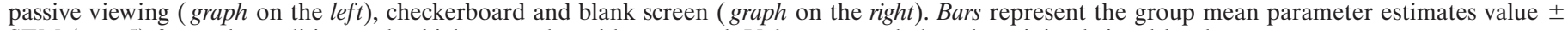
SEM $(n=5)$ for each condition under high, normal, and low arousal. Values are scaled to the minimal signal level.

the arousal system), the mediodorsal thalamic nucleus (considered the limbic relay for the prefrontal cortex), and the nucleus reticularis (which modulates the activity of the relay nuclei) are likely to be the target of top-down modulation of information flow, as suggested previously for other thalamic nuclei (La Berge, 1995; Frith and Friston, 1996). In particular the nucleus reticularis appears to be the best candidate for the interaction between arousal and attention because it receives axonal collaterals from the corticothalamic, thalamocortical, and midbrain reticular formation projection systems (Crick, 1984; Newman, 1995; Guillery et al., 1998) [stereotactic thalamic anatomy: Mai et al. (1997); functional thalamic anatomy: Jones (1985); Kelly (1991)].

This account suggests that the greater change in thalamic activity when subjects are performing the attentional task in a state of low arousal may reflect an enhancement of top-down modulation of multimodal inputs necessary to prevent the physiological shift of activity of the thalamocortical system toward incipient neuronal synchronization and the onset of sleep (Steriade et al., 1993).

This process may represent a sort of compensatory mechanism, a "window of activation" operating in extreme physiological conditions in which the arousal and attentional systems interact to modify the output of the thalamocortical system. We speculate that the thalamus has to "work harder" in conditions of low arousal to achieve a performance that is equal to that obtained during normal arousal.

We also consider that the systems involved in the visual processing and/or response selection required by the attentional task, but not by the passive viewing condition, may represent the targets of the attentional modulation exerted by the thalamus.

Finally, our findings support the role of the thalamus in the interplay between arousal and attention in humans as suggested previously by the study of Coull et al. (1997).

\section{Brain regions activated by attention but not affected by the level of arousal}

The brain regions that were activated by the attentional task but not affected by the level of arousal include areas of the posterior and anterior attentional networks (Posner and Dehaene, 1994). Previous positron emission tomography studies showed attentionrelated brain activation and left-right hemispheric functional asymmetry (Pardo et al., 1991; Bench et al., 1993; Jonides et al., 1993; Haxby et al., 1994; Petersen et al., 1994).

In the present study the bilateral activation of the posterior and superior parietal cortices and prestriate cortex are likely to be related to the processing of spatial-visual inputs (Mishkin et al., 1983). The activity in the anterior part of the cingulate sulcus and gyrus is consistent with the model of the "frontal midline atten- 
tional network" proposed by Goldman-Rakic and colleagues (Goldman-Rakic, 1988; Goldman-Rakic et al., 1993). The activation of the most anterior part of the middle frontal gyrus corresponding to the dorsolateral prefrontal cortex (BA 46) has been reported previously for the performance of tasks requiring attention and working memory (La Berge, 1995).

The persistent activation of these areas in the low arousal condition might be explained by increased thalamocortical activity maintaining a tonic level of neuronal excitation. This process may represent the higher "cost of attention" during low arousal that is subjectively experienced as mental effort. Such a compensatory mechanism might operate for a limited time only, explaining why a state of prolonged attention induces drowsiness (Babkoff et al., 1991). It is likely that increasing the duration of the sleep deprivation and/or the difficulty of the task beyond the parameters specified in this study would produce a decrease of the thalamocortical activity and of the cortical areas involved in the attentional response followed by a decrease in performance.

Finally, the bilateral activation of the premotor area (superior precentral sulcus and the most anterior part of the precentral gyrus) and cerebellum may be related to the anticipation of the motor response to the target (Stephan et al., 1995; Deiber et al., 1996). Attention-related activation of the cerebellum independent of motor involvement was reported recently (Allen et al., 1997).

\section{Control experiments}

The activation produced by the checkerboard stimulation did not show consistent changes across sessions in the experimental subjects or in the control subjects. This strongly suggests that hemodynamic changes were neither a function of arousal (or related to the experimental manipulation sleep deprivation/caffeine) nor of time.

In addition, in the control subjects there was no change in thalamic activation when performing the attentional task in day 1 , 2 , or 3 . This is consistent with our finding that greater thalamic activation occurs only when attention is applied in a state of lower arousal. The performance of the attentional task also produced a significant pattern of bilateral cortical activation that did not change consistently over the three sessions. This evidence further suggests the that there were no hemodynamic effects as a function of time.

\section{Conclusions}

The present data support the hypothesis that the thalamus represents the functional interface between the arousal and the attentional systems. The greater activation of the thalamic system when attention must be used during a state of low arousal may reflect a compensatory mechanism and may be related to the subjective experience of greater mental effort.

\section{REFERENCES}

Allen G, Buxton RB, Wong EC, Courchesne E (1997) Attentional activation of the cerebellum independent of motor involvement. Science 275:1940-1942.

Babkoff H, Caspy T, Mikulincer M (1991) Subjective sleepiness ratings: the effects of sleep deprivation, circadian rhythmicity and cognitive performance. Sleep 14:534-539.

Bench CJ, Frith CD, Grasby PM, Friston KJ, Paulesu E, Frackowiak RSJ, Dolan RJ (1993) Investigations of the functional anatomy of attention using the Stroop test. Neuropsychology 31:907-922.

Brendel DH, Reynolds CF, Jennings JR, Hoch CC, Monk TH, Berman SR, Hall FT, Buysse DJ, Kupfer DJ (1990) Sleep stage physiology, mood, and vigilance responses to total sleep deprivation in healthy 80-year-olds and 20-year-olds. Psychophysiology 27:677-685.
Bruce M, Scott N, Lader M, Marks V (1986) The psychopharmacological and electrophysiological effects of single doses of caffeine in healthy human subjects. Br J Pharmacol 22:81-87.

Buechel C, Friston KJ (1997) Principles and methods, statistical inference. In: Human brain function (Frackowiak RSJ, Friston KJ, Frith CD, Dolan RJ, Mazziotta JC, eds), pp 136-137. London: Academic.

Cameron OG, Modell JG, Hariharan M (1990) Caffeine and human cerebral blood flow: a positron emission tomography study. Life Sci 47:1141-1146.

Coull JT, Middleton HC, Robbins TW, Sahakian BJ (1995) Clonidine and diazepam have differential effects on tests of attention and learning. Psychopharmacology 120:322-332.

Coull JT, Frith CD, Dolan RJ, Frackowiak RSJ, Grasby PM (1997) The neural correlates of the noradrenergic modulation of human attention, arousal and learning. Eur J Neurosci 9:589-598.

Crick F (1984) Function of the thalamic reticular complex: the searchlight hypothesis. Proc Nat Acad Sci USA 81:4586-4590.

Das JP, Naglieri JA, Kirby JR (1994) Attention. In: Assessment of cognitive processes: the PASS theory of intelligence. pp 31-51. Boston: Allyn \& Bacon.

Deiber MP, Ibanez V, Sadato N, Hallett M (1996) Cerebral structures participating in motor preparation in humans: a positron emission tomography study. J Neurophysiol 75:233-247.

Easterbrook JA (1959) The effect of emotion on cue utilization and the organization of behavior. Psychol Rev 66:183-201.

Friston KJ, Worsley KJ, Frackowiak RSJ, Mazziotta JC, Evans AC (1994) Assessing the significance of focal activation using their spatial extent. Hum Brain Mapp 1:214-220.

Friston KJ, Holmes AP, Worsley KJ, Poline J-B, Frith CD, Frackowiak RSJ (1995) Statistical parametric mapping in functional imaging: a general linear approach. Hum Brain Mapp 2:189-210.

Friston KJ, Ashburner J, Poline J-B, Frith CD, Heather JD, Frackowiak RSJ (1996) Spatial realignment and normalization of images. Hum Brain Mapp 3:165-189.

Frith CD, Friston KJ (1996) The role of the thalamus in "top-down" modulation of attention to sound. NeuroImage 4:210-215.

Goldman-Rakic PS (1988) Topography of cognition: parallel distributed networks in primate association cortex. Annu Rev Neurosci 11:137-156.

Goldman-Rakic PS, Chafee M, Friedman H (1993) Allocation of function in distributed circuits. In: Brain mechanisms of perception and memory: from neuron to behavior (Ono T, Squire LR, Raichle ME, Perrett DI, Fukuda T, eds). New York: Oxford UP.

Guillery RW, Feig SL, Lozsadi DA (1998) Paying attention to the thalamic reticular nucleus. Trends Neurosci 21:28-32.

Harth E (1995) The sketchpad model, a theory of consciousness, perception and imagery. Consciousness Cogn 4:346-368.

Haxby JV, Horwitz B, Ungerleider LG, Maisog JM, Pietrini P, Grady CL (1994) The functional organization of human extrastriate cortex: a PET-rCBF study of selective attention to faces and locations. J Neurosci 14:6336-6353.

Heslegrave RJ, Angus RG (1985) The effect of task duration and worksession location on performance degradation induced by sleep loss and sustained cognitive work. Behav Res Methods Instrum Comput 17:592-603.

Holmes AP, Josephs O, Buchel C, Friston KJ (1997) Statistical modelling of low-frequency confounds in fMRI. NeuroImage 5:S480.

Jones ED (1985) The thalamus. New York: Plenum.

Jonides J, Smith EE, Koeppe RA, Awh E, Minoshima S, Mintun MA (1993) Spatial working memory in humans as revealed by PET. Nature 363:623-625.

Kahneman D (1973) Attention and effort. Englewood Cliffs, NJ: Prentice Hall.

Kelly JP (1991) The neural basis of perception and movement. In: Principles of neurosciences (Kandel ER, Schwartz JH, Jessell TJ, eds) Norwalk, CT: Appleton \& Lange.

Klerman EB, Dijk D-J, Kronauer RE, Czeisler CA (1996) Simulations of light effects on the human circadian pacemaker: implications for assessment of intrinsic period. Am J Physiol 270:R271-R282.

La Berge D (1995) Attentional processing. Cambridge, MA: Harvard UP.

La Berge D, Brown V (1989) Theory of attentional operations in shape identification. Psychol Rev 96:101-124. 
La Berge D, Buchsbaum MS (1990) Positron emission tomographic measurements of pulvinar activity during an attention task. J Neurosci 10:613-619.

La Berge D, Carter M, Brown V (1992) A network simulation of thalamic circuit operations in selective attention. Neural Comput 4:318-331.

Lynn R (1966) Attention, arousal and the orientation reaction. Oxford: Pergamon.

Luria AR (1973) The working brain. Harmondsworth, England: Penguin Books.

Mai JK, Assheuer J, Paxinos G (1997) Atlas of the human brain. San Diego: Academic.

McCormick DA, Feeser HR (1990) Functional implications of burst firing and single spike activity in lateral geniculate relay neurons. Neuroscience 39:103-113.

Mishkin M, Ungerleider LG, Macko KA (1983) Object vision and spatial vision: two cortical pathways. Trends Neurosci 6:414-417.

Newman J (1995) Thalamic contribution to attention and consciousness. Consciousness Cogn 4:172-193.

Olshausen BA, Anderson CH, Van Essen DC (1993) A neurobiological model of visual attention and invariant pattern recognition based on dynamic routing of information. J Neurosci 13:4700-4719.

Parasuraman R, Davies DR (1984) Varieties of attention. New York: Academic.

Pardo JV, Fox PT, Raichle ME (1991) Localization of a human system for sustained attention by positron emission tomography. Nature 349:61-64.

Petersen SE, Corbetta M, Miezin FM, Shulman GL (1994) PET studies of parietal involvement in spatial attention: comparison of different task types. Can J Exp Psychol 48:319-338.

Posner MI (1994) Attention: the mechanism of consciousness. Proc Natl Acad Sci USA 91:7398-7403.

Posner MI, Dehaene S (1994) Attentional networks. Trends Neurosci 17:75-79.

Price CJ, Moore CJ, Friston KJ (1997) Subtractions, conjunctions, and interactions in experimental design of activation studies. Hum Brain Mapp 5:264-272.
Rosenthal L, Merlotti L, Roehrs T, Roth T (1991a) Enforced $24 \mathrm{hr}$ recovery following sleep deprivation. Sleep 14:448-453.

Rosenthal L, Roehrs T, Zwyghuizen-Doorenbos A, Plath D, Roth T (1991b) Alerting effects of caffeine after normal and restricted sleep. Neuropsychopharmacology 4:103-108.

Stephan KM, Fink GR, Passingham RE, Silbersweig D, CeballosBaumann AO, Frith CD, Frackowiak RSJ (1995) Functional anatomy of the mental representation of upper extremity movements in healthy subjects. J Neurophysiol 73:373-386.

Steriade M, Contreras D, Curro' Dossi T, Nunez A (1993) The slow oscillation in reticular thalamic and thalamocortical neurons: scenario of sleep rhythm generation in interacting thalamic and neocortical networks. J Neurosci 13:3284-3299.

Talairach P, Tournoux J (1988) A stereotactic coplanar atlas of the human brain. New York: Thieme Verlag.

Tassi P, Nicolas A, Seegmuller C, Dewasmes G, Libert JP, Muzet A (1993) Interaction of the alerting effect of noise with partial sleep deprivation and the circadian rhythmicity of vigilance. Percept Mot Skills 77:1239-1248.

Turner R (1992) Magnetic resonance imaging of brain function. Am J Physiol Imaging 3/4:136-145.

Wilkinson RT (1965) Sleep deprivation. In: The physiology of human survival (Edholm OG, Bacharach AL, eds), pp 399-430. London: Academic.

Wilkinson RT (1968) Sleep deprivation: performance tests for partial and selective sleep deprivation. In: Progress in clinical psychology (Abt LA, Reiss BF, eds), pp 28-43. New York: Grune \& Stratton.

Wu JC, Gillin JC, Buchsbaum MS, Hershey T, Hazlett E, Sicotte N, Bunney WE (1991) The effect of sleep deprivation on cerebral glucose metabolic rate in normal humans assessed with positron emission tomography. Sleep 14:155-162.

Zwyghuizen-Doorenbos A, Roehrs TA, Lipschutz L, Timms V, Roth T (1990) Effects of caffeine on alertness. Psychopharmacology (Berl) 100:36-39. 